JAYYOSI, Charles, BRUYERE-GARNIER, Karine, CORET, Michel, 2017, Geometry of an inflated membrane in elliptic bulge tests: Evaluation of an ellipsoidal shape approximation by stereoscopic digital image correlation measurements, Medical Engineering \& Physics, 48, Supplement C, Elsevier, pp. 150-157, DOI: 10.1016/ j.medengphy.2017.06.020

\title{
Geometry of an inflated membrane in elliptic bulge tests: evaluation of an ellipsoidal shape approximation by stereoscopic digital image correlation measurements
}

\author{
C. Jayyosi ${ }^{1}$, K. Bruyère-Garnier* ${ }^{1}$ and M. Coret $^{2}$ \\ ${ }^{1}$ Univ Lyon, Université Claude Bernard Lyon 1, IFSTTAR, UMR_T9406, LBMC, F69622, Lyon, France ; \\ ${ }^{2}$ LUNAM Université, GEM, UMR CNRS 6183, Ecole Centrale de Nantes, Université de Nantes, France
}

Keywords: stereoscopic DIC; elliptic bulge test; ellipsoidal geometry; fibrous connective membrane; liver capsule

\begin{abstract}
Elliptic bulge tests are conducted on liver capsule, a fibrous connective membrane, associated with a field measurement method to assess the global geometry of the samples during the tests. The experimental set up is derived from a previous experimental campaign of bulge tests under microscope. Here, a stereoscopic Digital Image Correlation (DIC) system is used to measure global parameters on the test and investigate some assumptions made on the testing conditions which could not been assessed with microscopic measurements. In particular, the assumption of an ellipsoidal shape of the inflated membrane is tested by comparing the actual sample shape measured by stereoscopic DIC with an idealized ellipsoidal shape.

Results indicate that a rather constant gap exists between the idealized and actual position. The approximation in the calculation of a macroscopic strain through analytical modeling of the test is estimated here. The study of the liver capsule case shows that important differences can be observed in strain calculation depending on the method and assumptions taken. Therefore, analytical modeling of mechanical tests through ellipsoidal approximation needs to be carefully evaluated in every application. Here the field measurement allows assessing the validity of these modeling assumptions. Moreover, it gives precious details about the boundary conditions of the bulge test and revealed the heterogeneous clamping, highlighted by strain concentrations.
\end{abstract}

*Corresponding author: Karine Bruyère-Garnier, phone: +33472142368 , Email: karine.bruyere@ @ifsttar.fr, Address: 25 Avenue François Mitterrand 69675 Bron, France 


\section{Introduction}

Experimental protocols coupling microstructural observations to mechanical loading often require taking strong assumptions about the global geometry of the samples during the tests. It comes from the very local nature of the measurements, since it is often very complicated to combine simultaneous macroscopic and microscopic observations. However, in order to use the structural data obtained on a representative volume element (RVE), the local behavior identified has to be homogenized, and to do so, knowledge about the global behavior of the considered material is needed.

Therefore, in the case of bulge testing for instance, assumptions are made about the shape of the deformed sample to compute strain and stress from the measurement of the apex displacement [1-3]. The geometry is thus idealized in order to use the mathematical formulations developed for membranes, shells or plates [4,5].

An alternative to these assumptions is to use a field measurement method such as stereoscopic Digital Image Correlation (DIC). Stereoscopic DIC has often been used in the field of material characterization to perform field measurements at the macroscopic scale, because it allows obtaining information about the whole surface of a sample, and not local measurements of mechanical parameters that are usually obtained through sensors positioned in a specific location. Therefore, with these fields measurements, 2D strain fields can be obtained over the entire surface of the considered material from direct shape measurements and 2D stress fields can be computed numerically without any assumptions on geometrical symmetry [6,7]. DIC has thus intensively been used to study various biological materials. For instance, Tonge et al. [8] use a field measurement by stereocorrelation to study the skin anisotropy. Strain fields of the sclera [9], liver capsule [10], parenchyma [11,12] or skin [13] have also been investigated and obtained by DIC.

Therefore, the aim of this study is to use a stereoscopic DIC method to estimate the validity of the assumption of an idealized ellipsoidal deformed shape during an elliptic bulge test conducted on a thin connective membrane. To this end, we chose to adapt the bulge test set up presented in Jayyosi et al. [14] to perform a field measurement on the whole sample and thus, getting information on the macroscopic behavior, following the approach developed by Brunon $[10,15]$. This global measurement allows checking the hypotheses that are needed to perform an analytical modeling of the tests. These kind of modeling are required when the global field measurement is not accessible, for example in the case of experiments involving a more local imaging method than stereoscopic DIC as in Perrini et al. 2013 [16] or Jayyosi et al. 2015 [14].

\section{Material and Methods}

\section{Samples preparation}

The bulge tests have been performed on samples from 2 different human livers ( $\lesssim 86$ and $\delta 92$ years old), which have been harvested at the Department of Anatomy of the University of Rockefeller (DUAR). Informed consent of the subjects and ethical approval have been obtained since livers were harvested from subjects within the French voluntary corpse donation to science program. Following the same method used in Jayyosi et al. [14], circular samples of approximately 50mm diameter, have been drilled for the clamping screws in 3 different shape patterns ( $\mathrm{R} 1$ for circular samples, $\mathrm{R} 2$ for elliptic with a ratio 2 between major and minor axis, and R4 for elliptic samples with a ratio of 4). Figure 1a shows the dimensions of the test set up that determined the sizes of the samples. In total, 3 samples have been tested from liver \#1 (1xR1 1xR2 and 1xR4) and 8 from liver \#2 (3xR1, 3xR2 and $2 \times R 4)$. Samples from liver $\# 1$ were frozen at $-20^{\circ} \mathrm{C}$ then thawed at room temperature before testing. 
Samples from liver \#2 were tested fresh, i.e. within 3-4 days after the death, while being stored at $4^{\circ} \mathrm{C}$ on a tissue soaked with PBS to prevent dehydration.

DIC measurements are based on the analysis of random patterns on surfaces. Therefore, liver capsules have been spread over a silicone joint to be easily handle (figure 1b), and small drops of paint have been dropped off by gravity by spraying in parallel manner to the plane of the sample (figure 1c). Considering the sizes of the samples, the stains of the random pattern have to be very small to get a pattern heterogeneous enough to allow a good resolution for a precise computation on the whole area of observation. By spraying in a parallel manner to the sample, we can get rid of the large drops of paint that are ejected from the spray, and keep only the small drops of the paint mist, which drop off with gravity (figure 1c). Here, the average sizes of stains are in the order of 0.5-1 mm.

\section{Stereoscopic DIC set up}

The experimental set up previously developed for the bulge tests under microscope presented in Jayyosi et al. [14] have been reused. Briefly, after preparation, samples on silicone joints are clamped between a top ring and a bottom cylinder thanks to 8 clamping screws equally distributed around the inflation area, and the ensemble is placed into a PMMA (polymethyl methacrylate) inflation base (figure 2). As the liver capsule is translucent, the PMMA inflation base allows positioning the sample between the lighting and the camera. Thus, stereoscopic DIC is performed on the shadow of the random pattern projected on the cameras (figure 2). This particular set up allows avoiding problems of brightness or uniformity of lighting.

Two cameras (Fastcam SA3, Photron, Tokyo, Japan) have been used to record the test, each equipped with a 1024 x 1024 pixel $^{2}$ sensor. $105 \mathrm{~mm}$ macro objectives have been added to the cameras to adapt the field of view to our samples' dimensions. Depth of field has been set to be able to follow an out of plane displacement of $10 \mathrm{~mm}$, allowing recording the whole test (based on the indicative values obtained from the tests under microscope that indicated an out of plane displacement of 6-8 $\mathrm{mm}$ before failure). Cameras have been positioned to perform the correlation on the largest area on the sample, including the edges (figure 2). Then, a calibration has been performed thanks to a dedicated target to compute the relative position of the cameras, needed for the stereoscopic calculation.

The image acquisition has been performed at $50 \mathrm{~Hz}$ until failure to assess the rupture creation/propagation. Pressure was acquired at $100 \mathrm{~Hz}$ with accuracy of $\pm 1 \mathrm{mbar}$. As it was the case for the bulge tests under microscope, inflation is performed with compressed air. Loading is conducted with regular pressure increments up to failure, thanks to a manual valve.

\section{Data processing}

Displacement and Green-Lagrange strain fields are computed by the image correlation software VIC3D (Correlated Solutions, Inc.) [17]. Correlation is performed on a subset of 500-600 images equally distributed among all the images taken during the test, to keep a reasonable computation time. On these image sequences, the displacements are calculated on subsets of $31 \times 31$ pixel $^{2}$ wide and spaced of 7 pixels from one to another (figure 3a). A rotation of the computation basis has been applied to match with the basis linked to the sample (X axis on the major axis of the ellipse, $\mathrm{Y}$ axis on the minor axis of the ellipse and the $\mathrm{Z}$ axis corresponds to the vertical).

An analytical modeling of the inflated geometry is made for each test based on an approximation by an ellipsoid (equation 1). The dimensions of the approximated ellipsoid correspond to the dimensions of the ideal boundary conditions in the X Y plane (R1, R2 or R4), associated with the vertical position of the apex. 


$$
\left\{\begin{array}{c}
x=a \cos \theta \cos \varphi \\
y=b \cos \theta \sin \varphi \\
z=c \sin \theta
\end{array}\right.
$$

with $\theta \in[0, \pi]$ and $\varphi \in[-\pi, \pi]$, and where $a$ and $b$ are the dimension of the clamping in the XY plane (figure 1a), and $c$ the vertical position of the apex.

In order to test the validity of this approximation of the inflated dome geometry, the error between the real and approximated position of the capsule has been calculated, performing an orthogonal projection of the DIC measured surface on the idealized ellipsoidal surface. To do so, for each $i$ point of the DIC surface ( 2000-3000 points depending on the sample type), we look for the minimum distance $d_{i}$ with every $j$ points of the analytical surface (40000 points).

$$
d_{i}=\min _{j} \sqrt{\left(X_{\text {DIC } i}-X_{\text {Anal } j}\right)^{2}+\left(Y_{\text {DIC } i}-Y_{\text {Anal } j}\right)^{2}+\left(Z_{\text {DIC } i}-Z_{\text {Anal } j}\right)^{2}}
$$

Then a mean error is calculated at each pressure level as an average of the distance errors of each surface point.

In order to compare it to the DIC strain, macroscopic strain derived from the deformed ellipsoid (referred to as ellipsoidal strain in the following) is also computed based on that ellipsoid geometry assumption. The ellipsoid meridian length in $\mathrm{X}$ and $\mathrm{Y}$ directions are calculated from the second formula of Ramanujan [18], which gives for the $\mathrm{X}$ direction:

$$
L_{x} \approx \frac{\pi(c+a)}{4}\left[1+\frac{3 h}{10+\sqrt{4-3 h}}\right]
$$

where

$$
h=\left(\frac{c-a}{c+a}\right)^{2}
$$

where $a$ and $c$ are the previously mentioned dimensions of the ellipsoid ( $a$ is replaced by $b$ in this formula to calculate the meridian length in the $\mathrm{Y}$ direction).

Then, the stretch ratios in $\mathrm{X}$ and $\mathrm{Y}$ directions are given by:

$$
\left\{\begin{array}{l}
\lambda_{x}=\frac{L_{x}}{a} \\
\lambda_{y}=\frac{L_{y}}{b}
\end{array}\right.
$$

And Green-Lagrange strain at the apex in $\mathrm{X}$ and $\mathrm{Y}$ directions is calculated as follow:

$$
\left\{\begin{array}{l}
E_{x x}=\frac{1}{2}\left(\lambda_{x}{ }^{2}-1\right) \\
E_{y y}=\frac{1}{2}\left(\lambda_{y}{ }^{2}-1\right)
\end{array}\right.
$$




\section{Results}

\section{Evolution of the apex vertical displacement with pressure increase}

The initial state for image correlation has been chosen to correspond with the state where the capsule presents a uniform curvature, which is achieved for a pressure of $0.02 \mathrm{bar}$, in accordance with what was previously reported by Brunon [15]. This initial pressure of $0.02 \mathrm{bar}$ is almost 20 times smaller compared to rupture pressure.

An example of the global shape of the inflated capsule is given on figure 4. We notice that the correlation does not work systematically on the edges near the clamping due to small droplets of saline solution which can gather up in these areas and erase the random pattern locally, making the correlation fail. However, the particular positioning of the cameras allows keeping a large area for the correlation computation, as the left and right cameras show a large common area in their respective field of view (figure 4).

The evolution of the vertical displacement of the apex with the pressure increase presents some variability depending on the sample considered. Figure 5 shows the apex vertical displacementpressure curves. The sudden increase in vertical displacement for sample \#1 at the end of the test comes from slippage of the capsule in the clamping. Globally speaking, the specific geometry of the different test types induces a greater vertical displacement for the circular samples than for the elliptic ones.

\section{Geometry approximation by an ellipsoid}

Figure 6a illustrates the evolution of the mean error between the shape measured by stereoscopic DIC, and the idealized ellipsoidal shape. This error is within the range of 0.25-0.4 mm and does not vary much with the level of pressure (except for a few cases). Figure $6 \mathrm{~b}$ gives an example of the standard deviation associated to the mean errors computed over the all surface area for a sample of each type $\mathrm{R} 1, \mathrm{R} 2$ and R4. The standard deviation appears quite important as the error between the position of the two surfaces varies depending on the location on the ellipsoid (it is null at the apex and increase as we get closer from the outer perimeter). An example of the comparison between idealized and real shapes is given on figure 7 .

\section{Stereoscopic DIC strain vs ellipsoidal strain}

Post processing reveals a mean deformation rate around $2.10^{-3} \mathrm{~s}^{-1}$ in the $\mathrm{Y}$ direction (which correspond to the direction of the minor axis of the ellipses).

Figure 8 presents the comparison between the stereoscopic DIC strain and the ellipsoidal strain at the apex in the $\mathrm{X}$ and $\mathrm{Y}$ directions, for all the samples tested. A distinction is made between the different sample types R1, R2 and R4. An important gap can be observed between the two calculated strains over the samples considered, particularly for the R4 samples. For the R1 samples the stereo and ellipsoidal strains match properly except for the first sample (which experienced sliding at the end as seen on figure 5).

\section{Strain fields}

Figure 9 shows an example of the Green-Lagrange strain fields $E_{x x}$ and $E_{y y}$ as well as principal strain $E_{1}$ and $E_{2}$ for each sample type. Strain fields often show some strain concentrations around the boundaries at the clamping. The non-uniformity of the tightening of the clamping screws induces heterogeneities in the strain fields. However, these variations are successfully assessed by the field measurements by stereoscopic DIC, which allows getting a realistic idea of the effective strain state of the sample. It therefore gives an accurate assessment of the real boundary conditions of the bulge test. We notice that the directions of principal strain for the elliptic samples match properly the $\mathrm{X}$ and $\mathrm{Y}$ 
directions of the ellipse when the tightening is uniform (figure 9b). On the other hand, these directions can differ from the directions of the ellipse when the tightening induces heterogeneities in the strain fields (figure 9c).

\section{Discussion}

This experimental campaign aim was to quantify at the macroscopic scale the loading conditions applied during the experiments under microscope described in Jayyosi et al. [14]. The displacement field measurements on the whole sample allowed assessing the real sample geometry and boundary conditions during the bulge test.

The relative error between the real position of the capsule and the position approximated by an ellipsoid indicates that the analytical modeling of the test must be carried out carefully. Using ellipsoid parametric equations, the stretch ratios can be computed to estimate a global strain measure on the different tests from the apex displacement value solely. However, the error on the position of the capsule made with the ellipsoidal assumption induces uncertainty on the macroscopic strain calculation based on such analytical modeling. Indeed, a mean error of $0.35 \mathrm{~mm}$ on the apex position, which is globally observed on the samples tested here as seen on figure 6 , should create an uncertainty between $0-5 \%$ on the macroscopic strain value calculated. Therefore, the estimation of macroscopic strain from that perspective remains an approximation.

In fact, the difference between stereoscopic strain and ellipsoidal strain exceed those $5 \%$ strain to reach $10-15 \%$ in extreme cases as seen on figure 8 . The additional assumptions taken to calculate strain (especially in the stretch ratios calculation) combined with the uncertainty on the apex position lead to significant errors on ellipsoidal strain estimation.

It is noteworthy that here, the ellipsoidal approximation made is not optimal as the DIC surface has not been fit with an ellipsoid with the objective of minimizing the error between the two surfaces. Figure $6 \mathrm{~b}$ highlights this point by showing the important standard deviation of errors over the all surface. The aim was to compare the measured DIC shape with an ellipsoidal approximation made solely from the knowledge of the apex displacement and the boundary conditions, to evaluate the analytical modeling of the test based on these scarce measurements. A more accurate analytical modeling of the test could be perform from the data of the field measurements by looking for an optimal ellipsoidal fit as in $[8,10]$.

Another source of explanation for the difference observed between the deformed shape and the analytical shape could come from the heterogeneity or the anisotropy of the material. Indeed, Tonge et al. [8] showed that skin anisotropy induce an ellipsoidal shape for the inflated sample with circular boundary conditions instead of a spherical shape. Such effect cannot be predicted with the kind of analytical modeling presented here. However, for the case of liver capsule, this effect is not likely to have a significant impact as the microstructural organization of this tissue does not present a clear marked anisotropy [14], and the optimized spherical fit of a bulge test of liver capsule from DIC measurements gave satisfying results [10].

A high variability of behavior among the different samples is observed, as seen on figure 5 . It highlights the difficulty of identifying mechanical properties for biological tissues. Indeed, the intersamples variability within one liver is already important. To that variability, it must be added the dispersion that comes from the inter-individual variability, which makes it even more difficult to identify a unique law to describe the mechanical behavior. A part of the variability observed here also comes from the specific testing conditions. The loading rate is not completely identical from one sample to another, despite the experimental care to make every test repeatable. Thus, the tests comparison is made without any considerations to viscous effects. 
Moreover, the clamping realized by screws equally distributed around the sample is not strictly uniform, and probably has an effect on the displacements measured by stereoscopic DIC (this is the case for sample \#1 for example that slipped at the end of the test). This non uniform clamping induces a non-homogeneous deformation of the silicone joint which creates the heterogeneities in the boundary conditions. The problem mentioned in the Brunon thesis [15], about the boundary conditions control by realizing a precise and uniform clamping has not entirely been solved here. Positioning more clamping screws allowed limiting slippage of the sample, by handling more precisely the boundary conditions. However, the field measurement still reveals the existence of heterogeneous strain concentrations on the borders of the samples, as highlighted by figure 9. These stress concentrations in the clamping screws question one of the perks of bulge testing, which is to load the sample far from the edges, to avoid rupture initiation due to cutting. Still, it is noteworthy that the field measurement allows assessing and quantifying this phenomenon, and by doing so, knowing the actual strain state of the material during the test.

The heterogeneity of the strain fields also points out a limitation of the ellipsoidal analytical modeling of the test since these disparities in strain cannot be accounted for with such modeling. Therefore, the gap between stereoscopic DIC strain and ellipsoidal strain, seen on figure 8, comes also from this phenomenon. This kind of analytical modeling based on scarce measurements does not take into account the heterogeneous boundary conditions. In that regard, field measurements appear as a valuable and practical solution when a detailed knowledge of the all sample mechanical state is needed. However, when such methods as stereoscopic DIC cannot be used, the alternative methods and hypotheses taken to infer information from scarcer measurements need be to carefully investigated to get a clear idea on how they impact the obtained results [19].

\section{Conclusion}

The implementation of a macroscopic field measurements coupled to the bulged tests, previously developed for microscopic tests, allowed accurately assessing the experimental conditions of the inflation test. Especially, the global geometry of the inflated dome and the boundary conditions during the test have been measured, thanks to the stereoscopic DIC method.

Stress concentrations occurred on the edges during the inflation, thus revealing a limitation of the experimental protocol, associated with the non-uniform clamping of the sample.

The field measurement by stereoscopic DIC offers the possibility to evaluate the performance of the ellipsoidal shape assumption. This assumption is necessary to compute macroscopic strain from scarce measurements, which is the case in the microscopic tests performed under microscope, where just the measure of the apex displacement is made. The evaluation of this analytical modeling performance provides knowledge about the accuracy of this macroscopic strain estimation. Eventually, the comparison between macroscopic and microscopic strains will allow linking the microstructural properties to the macroscopic behavior of the liver capsule.

\section{Disclosure}

None of the authors have any professional or financial conflict of interest.

\section{Acknowledgments}


This work was supported by the Programme Avenir Lyon Saint-Etienne (ANR-11-IDEX-0007) of Université de Lyon, within the program "Investissements d'Avenir" operated by the French National Research Agency (ANR).

\section{References}

[1] Drexler ES, Quinn TP, Slifka a J, McCowan CN, Bischoff JE, Wright JE, et al. Comparison of mechanical behavior among the extrapulmonary arteries from rats. J Biomech 2007;40:812-9. doi:10.1016/j.jbiomech.2006.03.010.

[2] Yousif MI, Duncan JL, Johnson W. Plastic deformation and failure of thin elliptical diaphragms. Int J Mech Sci 1970;12:959-IN2. doi:10.1016/0020-7403(70)90036-6.

[3] Rees DW. Plastic flow in the elliptical bulge test. Int J Mech Sci 1995;37:373-89. doi:10.1016/0020-7403(94)00071-Q.

[4] Flügge W. Stresses in Shells. Springer Berlin Heidelberg; 1960. doi:10.1007/978-3-662-010280 .

[5] Feng WW. Viscoelastic Behavior of Elastomeric Membranes. J Appl Mech 1992;59:S29-34.

[6] Davis FM, Luo Y, Avril S, Duprey A, Lu J. Pointwise characterization of the elastic properties of planar soft tissues: application to ascending thoracic aneurysms. Biomech Model Mechanobiol 2015;14:967-78. doi:10.1007/s10237-014-0646-9.

[7] Genovese K, Casaletto L, Humphrey JD, Lu J. Digital image correlation-based point-wise inverse characterization of heterogeneous material properties of gallbladder in vitro. Proc $\mathrm{R}$ Soc London A Math Phys Eng Sci 2014;470.

[8] Tonge TK, Atlan LS, Voo LM, Nguyen TD. Full-field bulge test for planar anisotropic tissues: part I--experimental methods applied to human skin tissue. Acta Biomater 2013;9:5913-25. doi:10.1016/j.actbio.2012.11.035.

[9] Myers KM, Coudrillier B, Boyce BL, Nguyen TD. The inflation response of the posterior bovine sclera. Acta Biomater 2010;6:4327-35. doi:10.1016/j.actbio.2010.06.007.

[10] Brunon A, Bruyère-Garnier K, Coret $\mathrm{M}$. Characterization of the nonlinear behaviour and the failure of human liver capsule through inflation tests. J Mech Behav Biomed Mater 2011;4:1572-81. doi:10.1016/j.jmbbm.2010.12.016.

[11] Brunon A, Bruyère-Garnier K, Coret M. Mechanical characterization of liver capsule through uniaxial quasi-static tensile tests until failure. J Biomech 2010;43:2221-7. doi:10.1016/j.jbiomech.2010.03.038.

[12] Gao Z, Desai JP. Estimating zero-strain states of very soft tissue under gravity loading using digital image correlation. Med Image Anal 2010;14:126-37. doi:10.1016/j.media.2009.11.002.

[13] Jacquemoud C, Bruyere-Garnier K, Coret M. Methodology to determine failure characteristics of planar soft tissues using a dynamic tensile test. J Biomech 2007;40:468-75. doi:10.1016/j.jbiomech.2005.12.010.

[14] Jayyosi C, Coret M, Bruyère-Garnier K. Characterizing liver capsule microstructure via in situ bulge test coupled with multiphoton imaging. J Mech Behav Biomed Mater 2016;54:229-43. 
doi:10.1016/j.jmbbm.2015.09.031.

[15] Brunon A. Caractérisation et modélisation de la rupture des tissus hépatiques. 2011.

[16] Perrini M, Bürzle W, Haller C, Ochsenbein-Kölble N, Deprest J, Zimmermann R, et al. Contractions, a risk for premature rupture of fetal membranes: a new protocol with cyclic biaxial tension. Med Eng Phys 2013;35:846-51. doi:10.1016/j.medengphy.2012.08.014.

[17] Sutton M, Orteu J, Schreier H. Image correlation for shape, motion and deformation measurements. 2009. doi:10.1007/978-0-387-78747-3.

[18] Ramanujan S. Modular equations and approximations to pi. Q J Math 1914;45:350-72.

[19] Murienne BJ, Nguyen TD. A comparison of 2D and 3D digital image correlation for a membrane under inflation. Opt Lasers Eng 2016;77:92-9. doi:10.1016/j.optlaseng.2015.07.013.

\section{Figure legends}

Figure 1: a) Dimensions of the bulge test set up: $R 1$ : $a=b=10 \mathrm{~mm}, R 2$ : $a=12 \mathrm{~mm}$ and $b=6 \mathrm{~mm}, \mathrm{R} 4$ : $a=20 \mathrm{~mm}$ and $b=5 \mathrm{~mm}$. b) Samples spread over the silicone joint on which we perform the random pattern for stereoscopic DIC. c) Random pattern realization technique with drop off of paint drops by gravity.

Figure 2: Lay out of the experimental set up with the PMMA inflation base positioned between the lighting spots and the 2 cameras for stereoscopic correlation.

Figure 3: a) Initial subset of the image showing the random pattern before deformation. b) Deformed subset with updated position (VIC3D®)

Figure 4: Shapes of the inflated liver capsules for the 3 test types R1 (circular), R2 (elliptic with ratio 2 between minor and major axis) and R4 (elliptic with ratio 4), associated with the images of the left and right cameras.

Figure 5: Evolution of vertical displacement of the liver capsule apex with pressure increase, for the different types of loading considered (R1, R2 and R4).

Figure 6: a) Mean error between the actual position measured by stereoscopic DIC and the approximated position of the capsule by an ellipsoid. The approximated ellipsoid is based on the theoretical dimensions of the clamping and the vertical displacement of the apex. b) Standard deviation associated to the error calculated over the all surfaces for sample \#3 (R1), \#7(R2) and \#9 (R4).

Figure 7: Geometry of the inflated capsule for a R4 sample (blue) put in relation with the ellipsoidal approximation (red).

Figure 8: Comparison between stereoscopic DIC strain (-) and ellipsoidal strain (..) in the X direction (left column) and Y direction (right column), for all the samples tested (sorted out between R1, R2 and R4). 
Figure 9: Example of Green-Lagrange strain fields in the $\mathrm{X}, \mathrm{Y}$ and principal directions for a $\mathrm{R} 1$ sample (a), R2 sample (b) and R4 sample (c).

\section{Figure 1a}

\section{a}

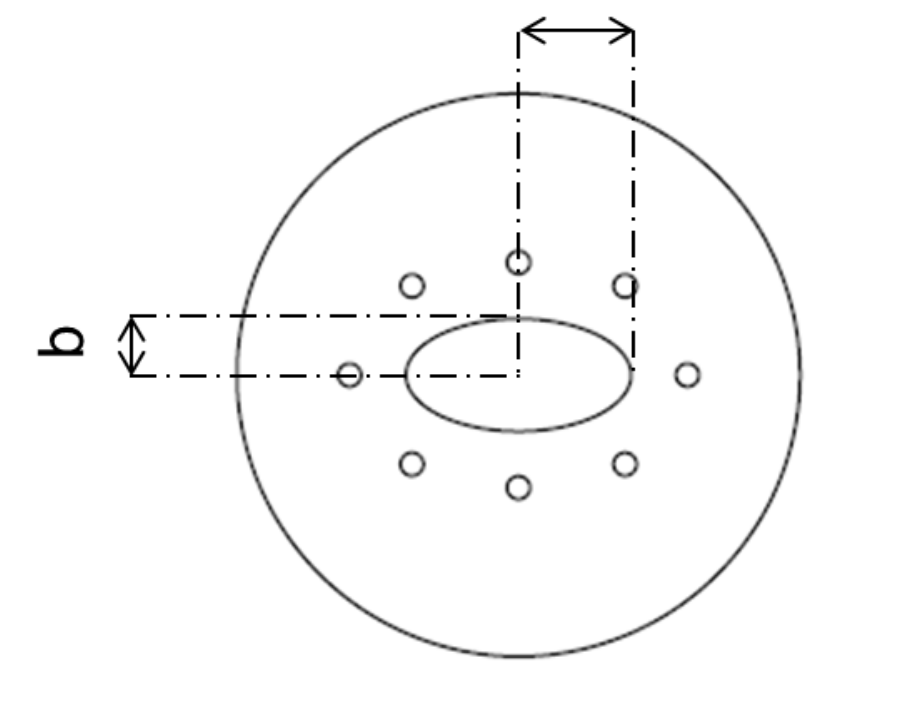

Figure $1 b$

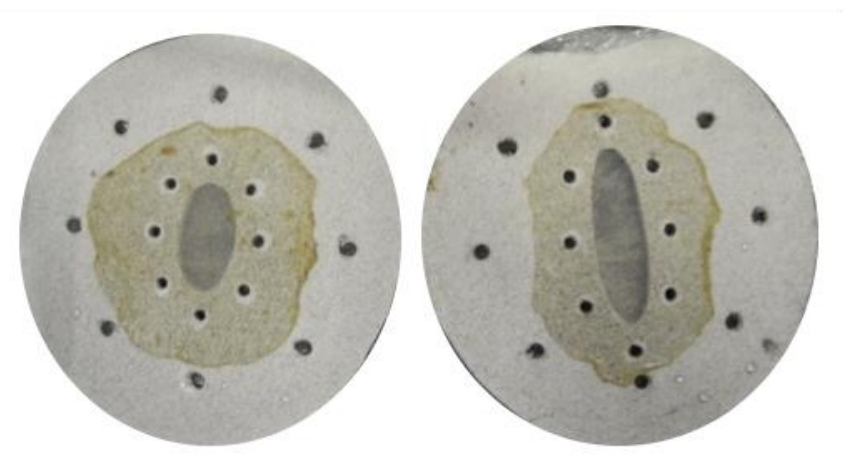


Figure 1c

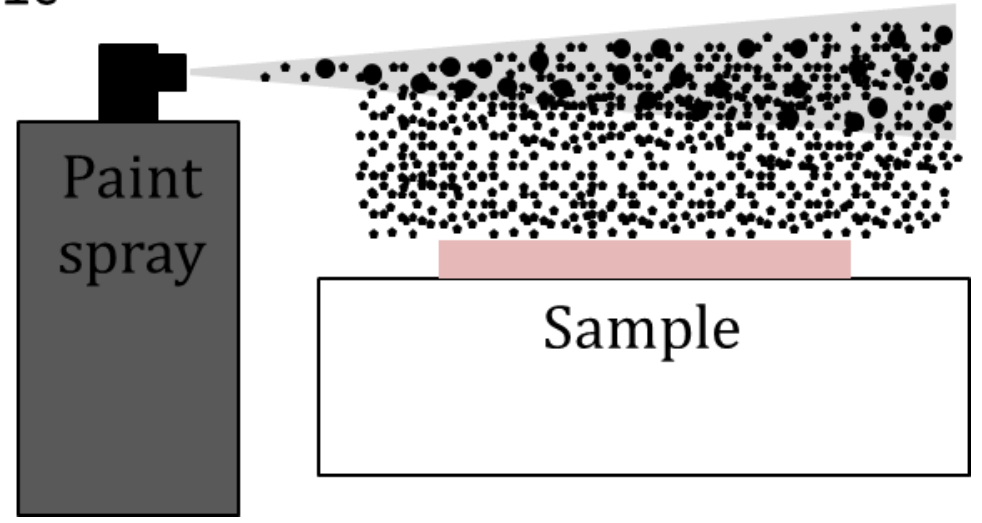

Figure 2
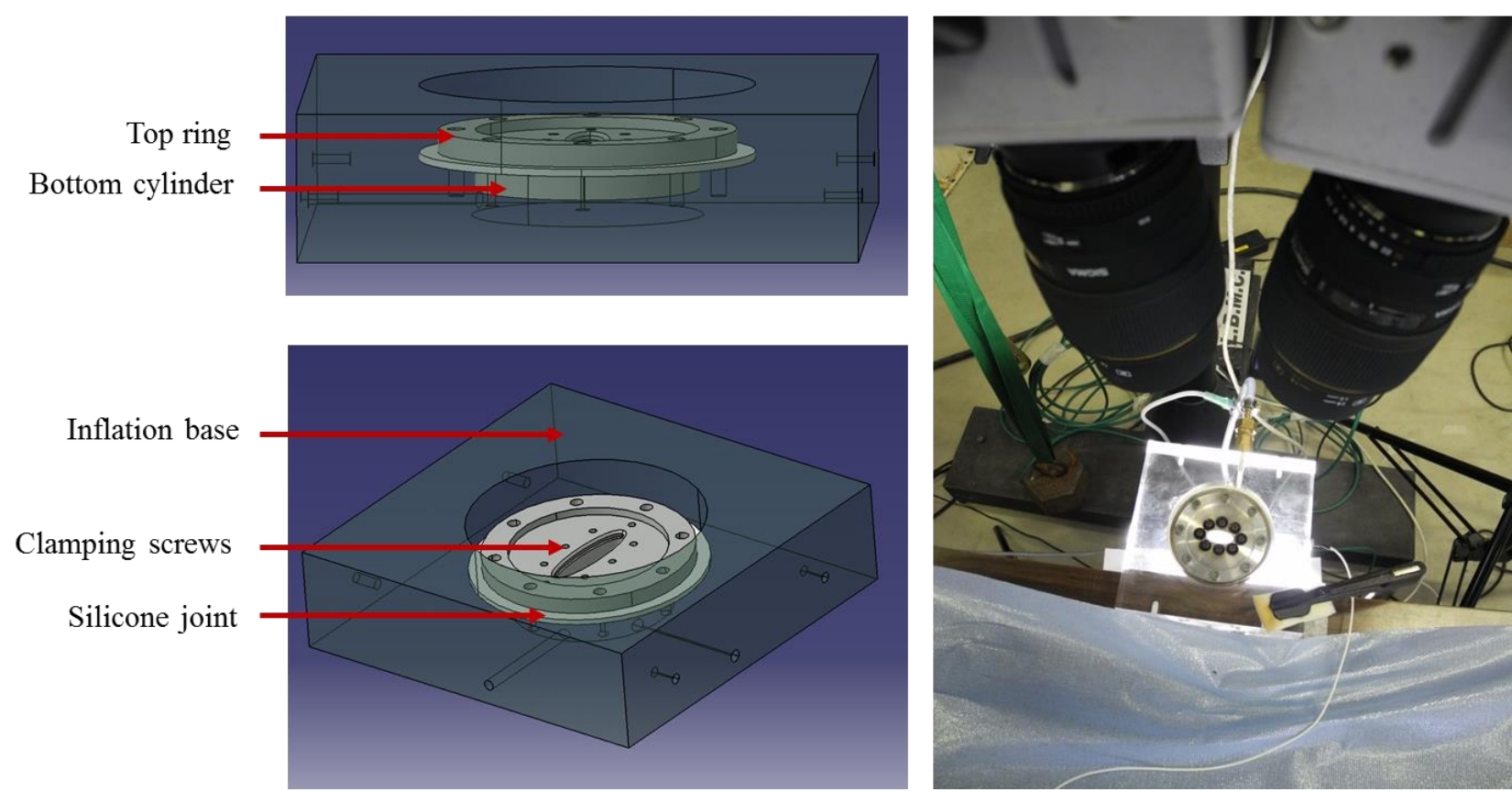
Figure 3a

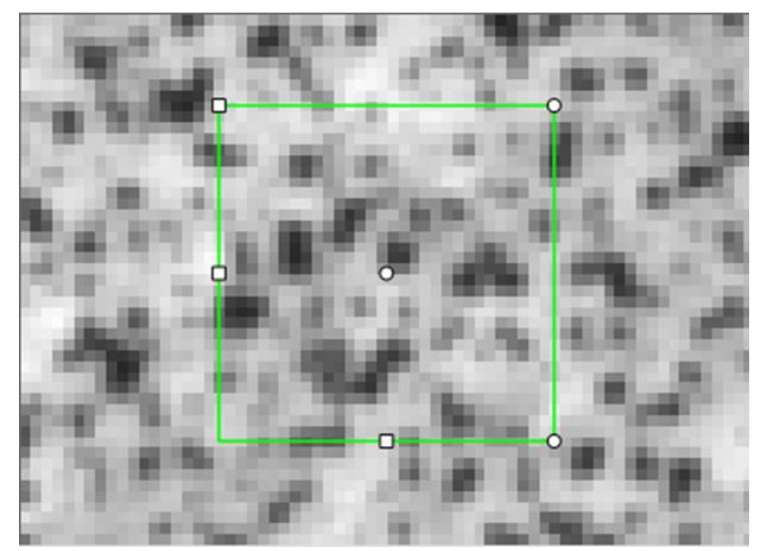

Figure 3b

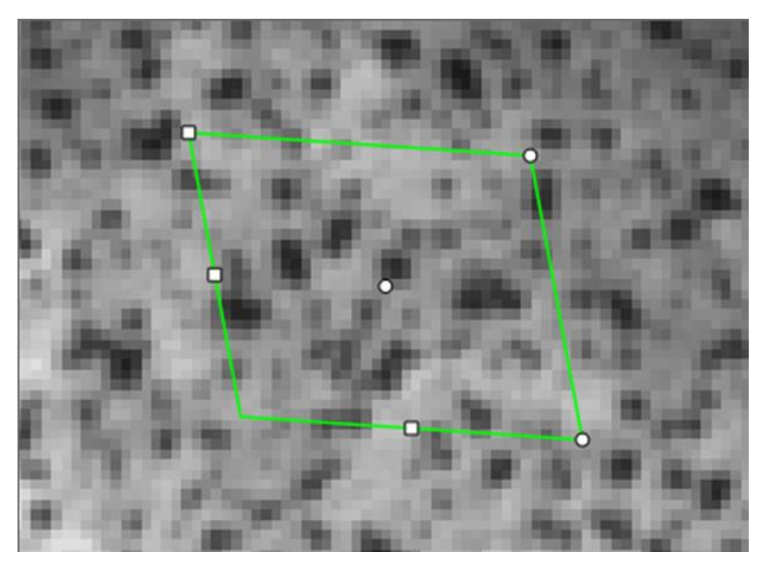

Figure 4
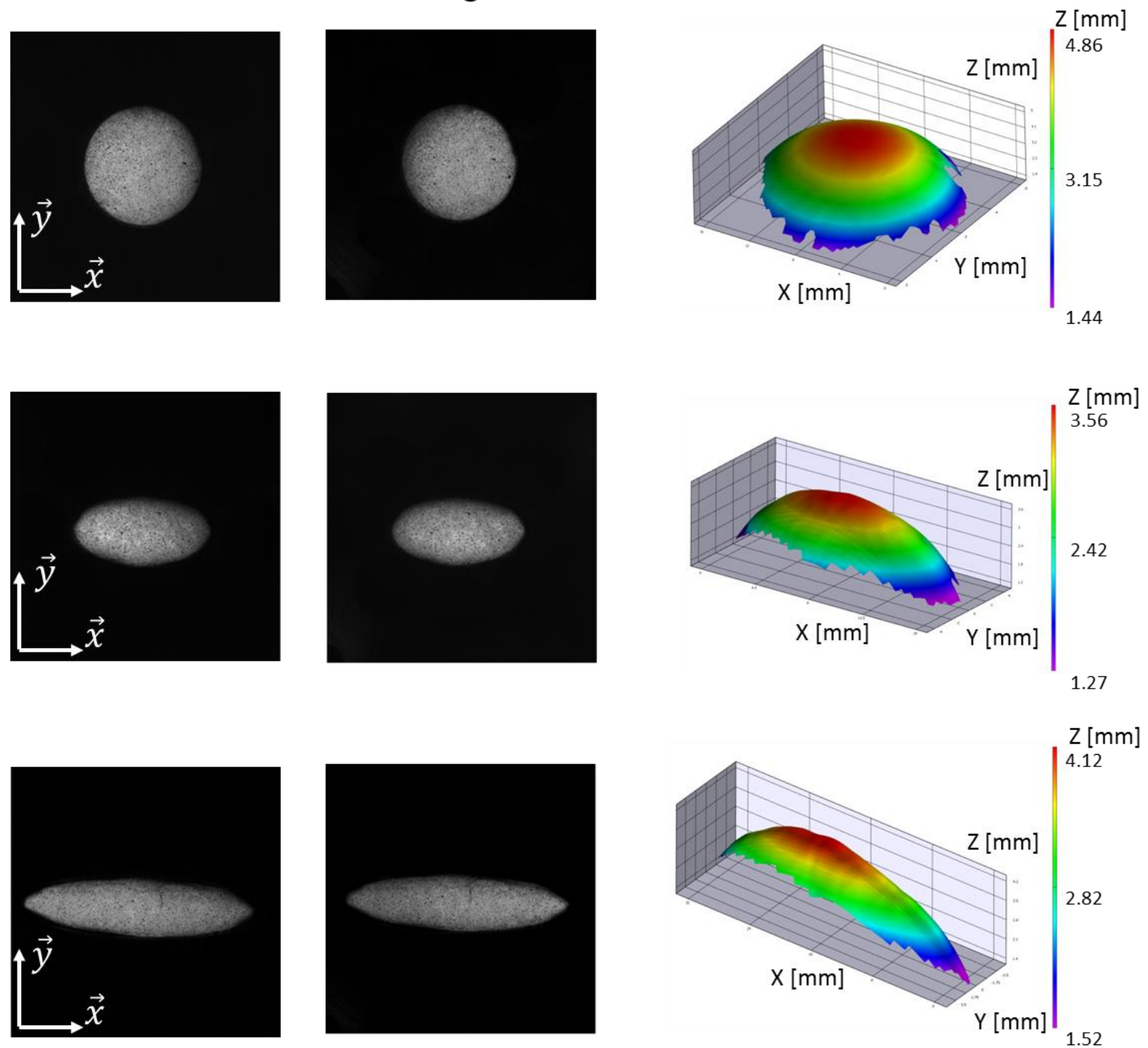
Figure 5

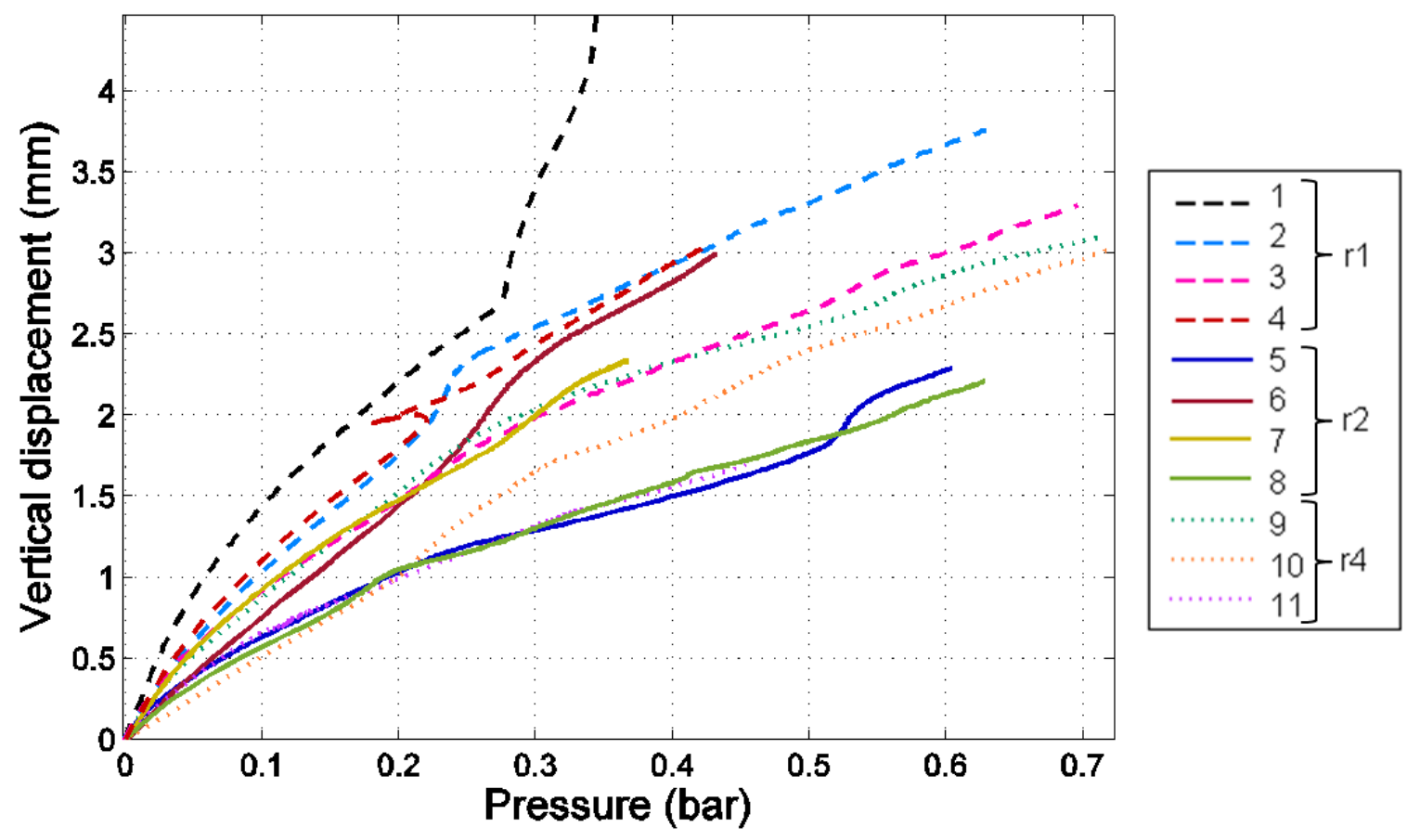

Figure 6a

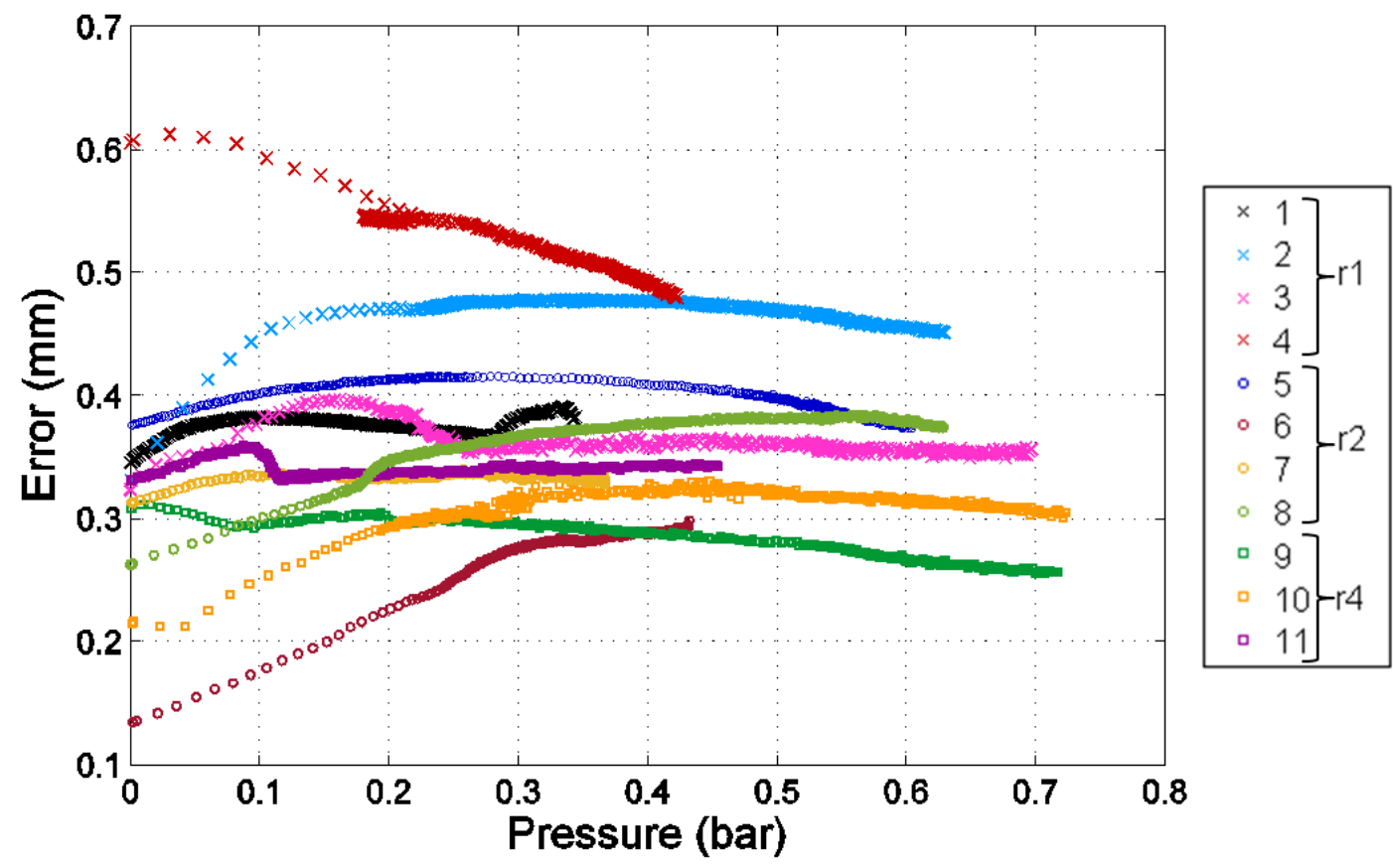


Figure $6 b$

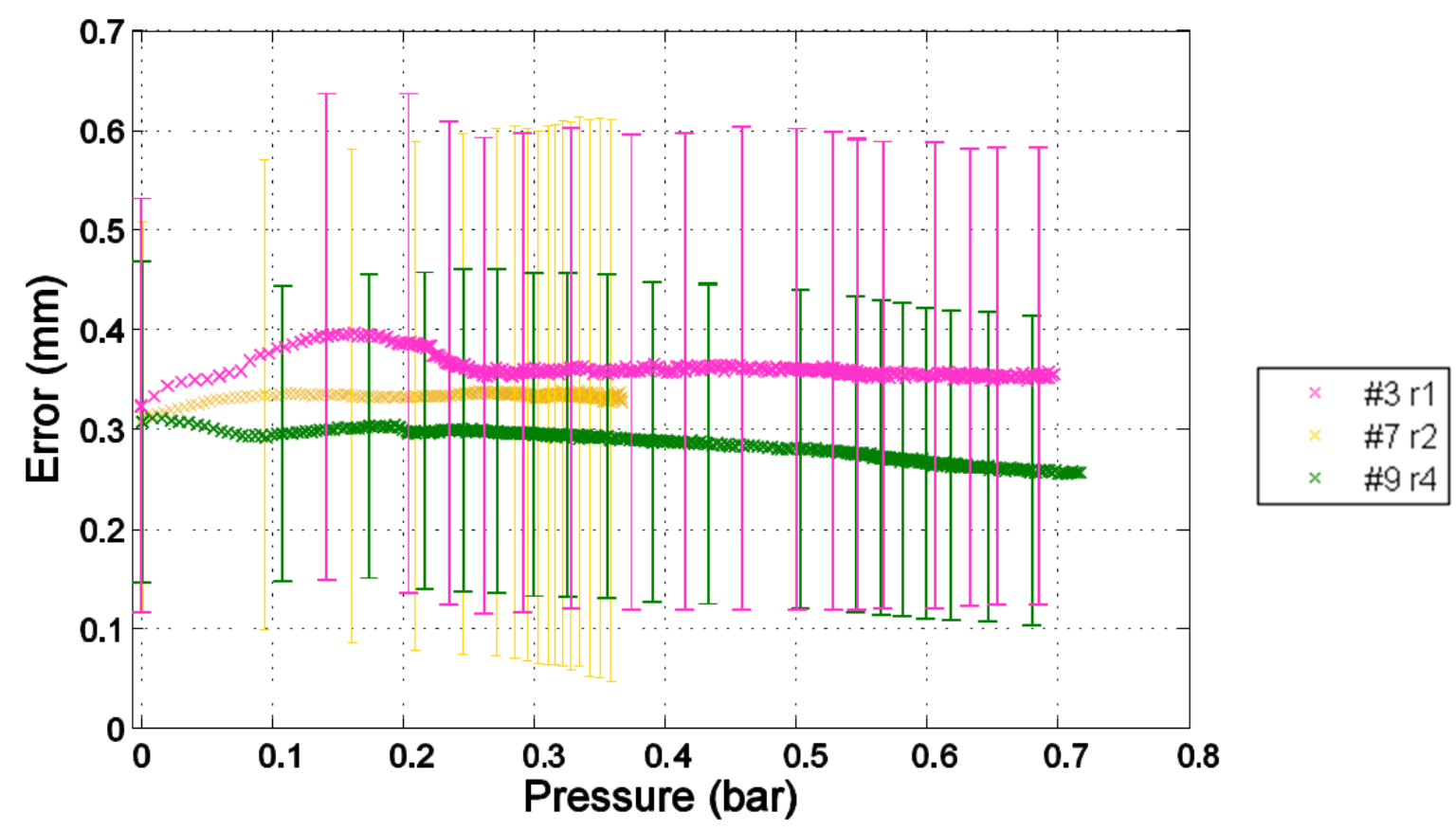


Figure 7
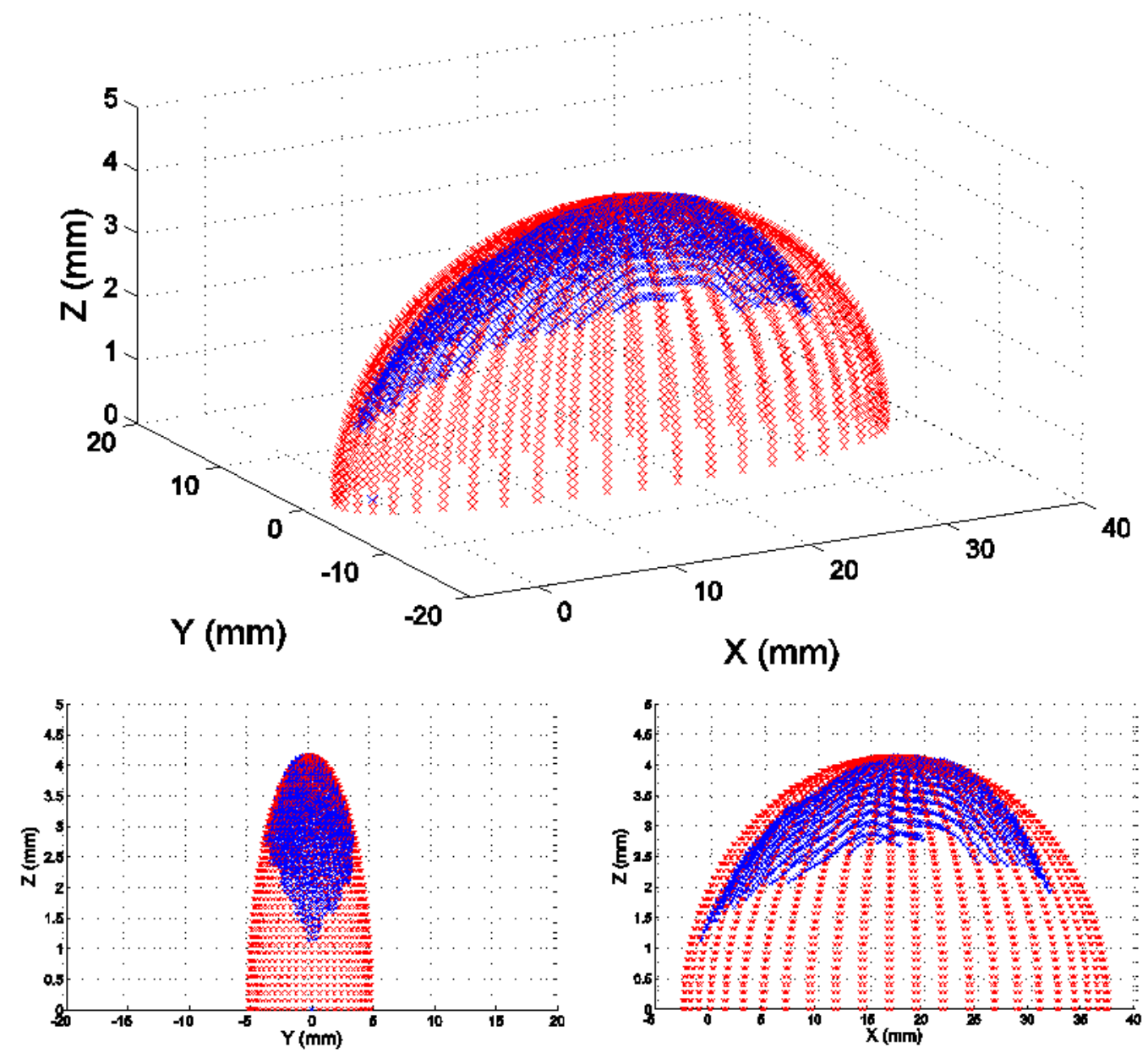
Figure 8
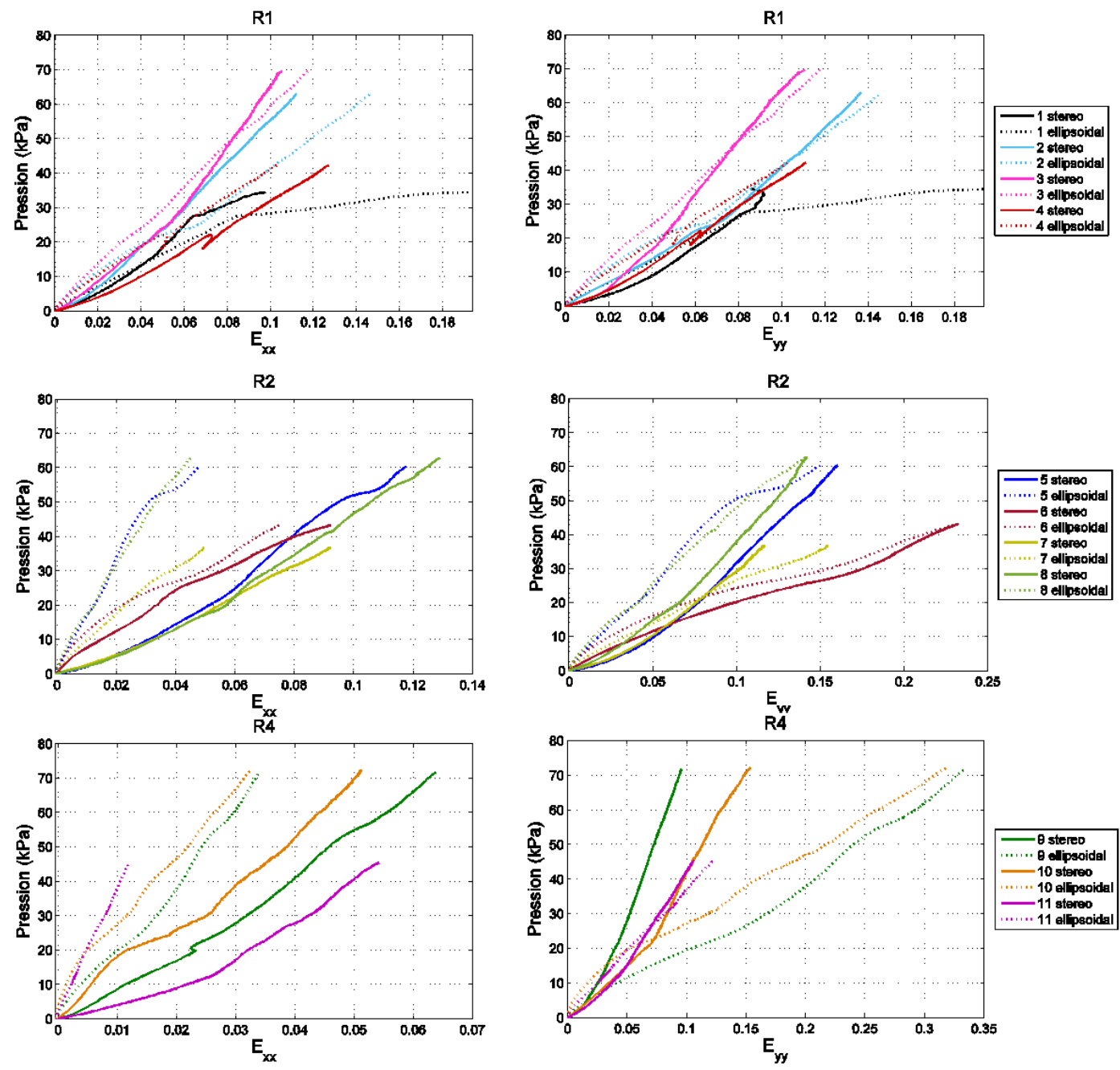
a)
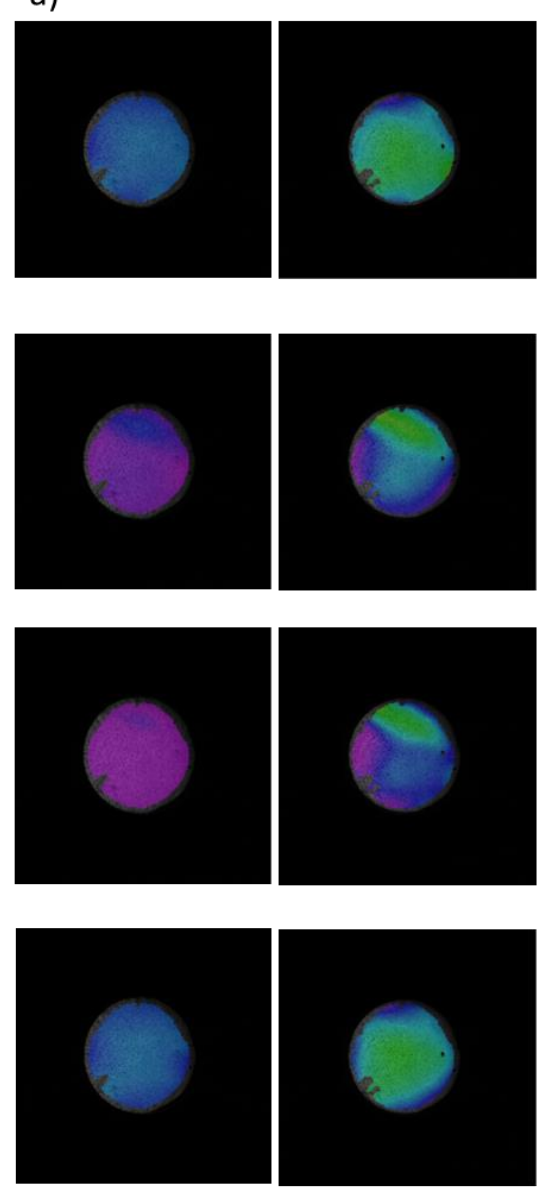

Figure 9
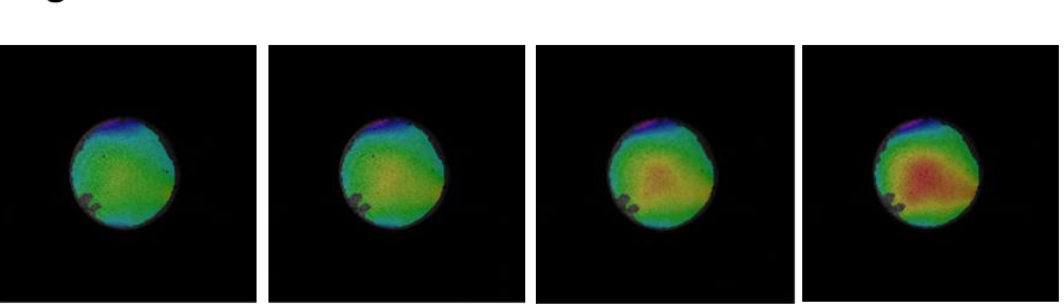

$\mathrm{E}_{\mathrm{xx}} 0.12$

0.12

0.045

$-0.03$
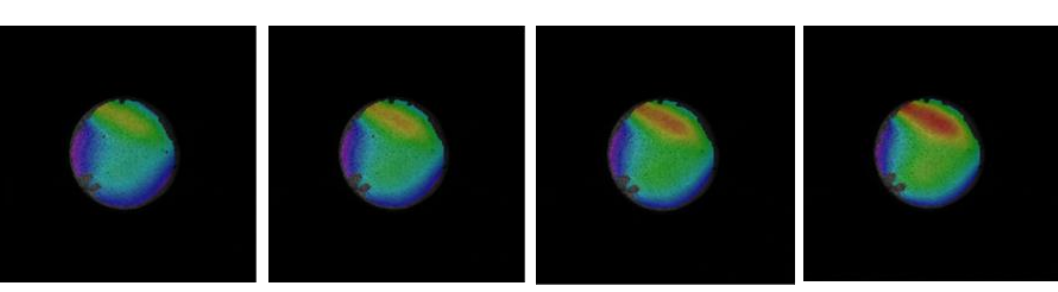

$\mathrm{E}_{\mathrm{yy}}$

0.21

0.105

$E_{1} 0$
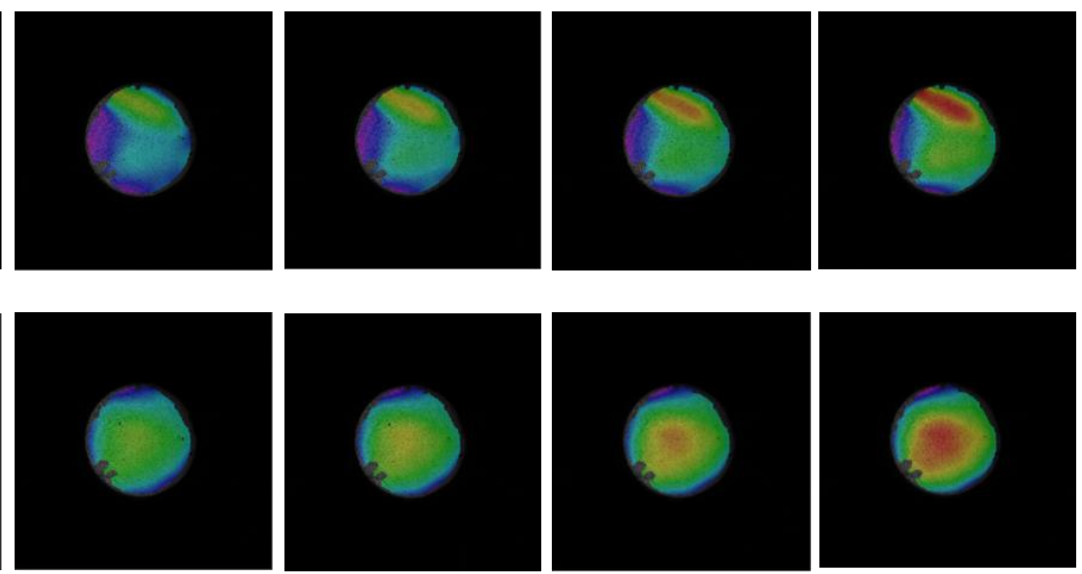

$\mathrm{E}_{2} 0.03$

0.11 
Figure 9

b)
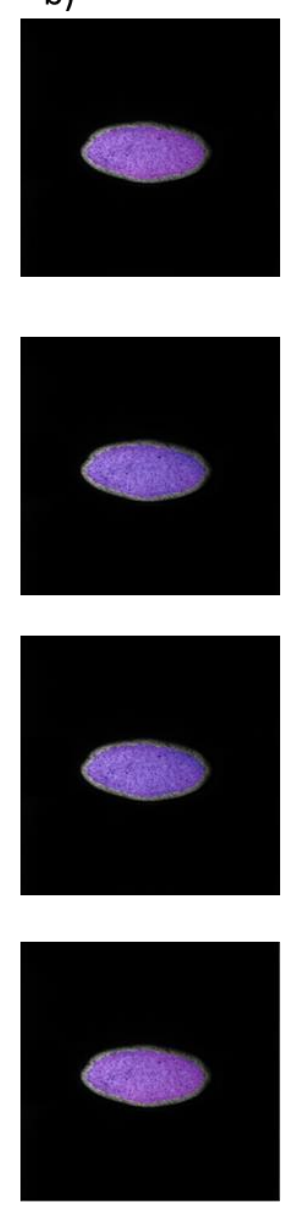
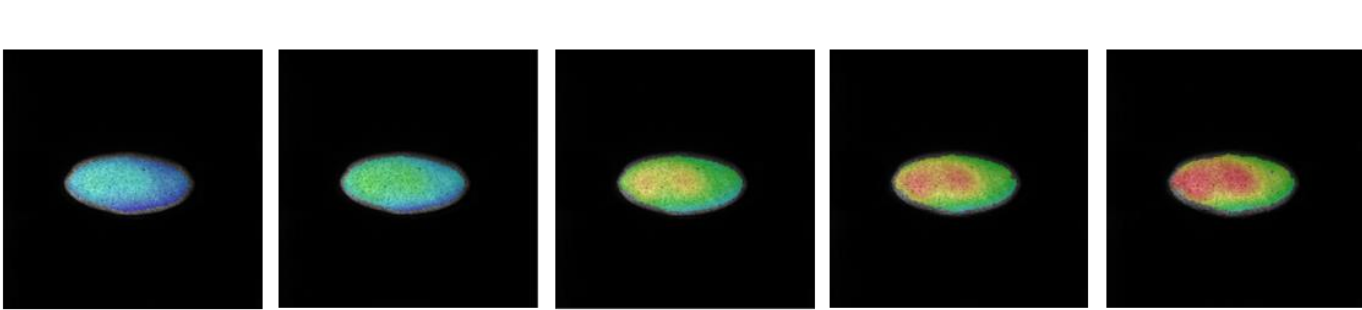

E
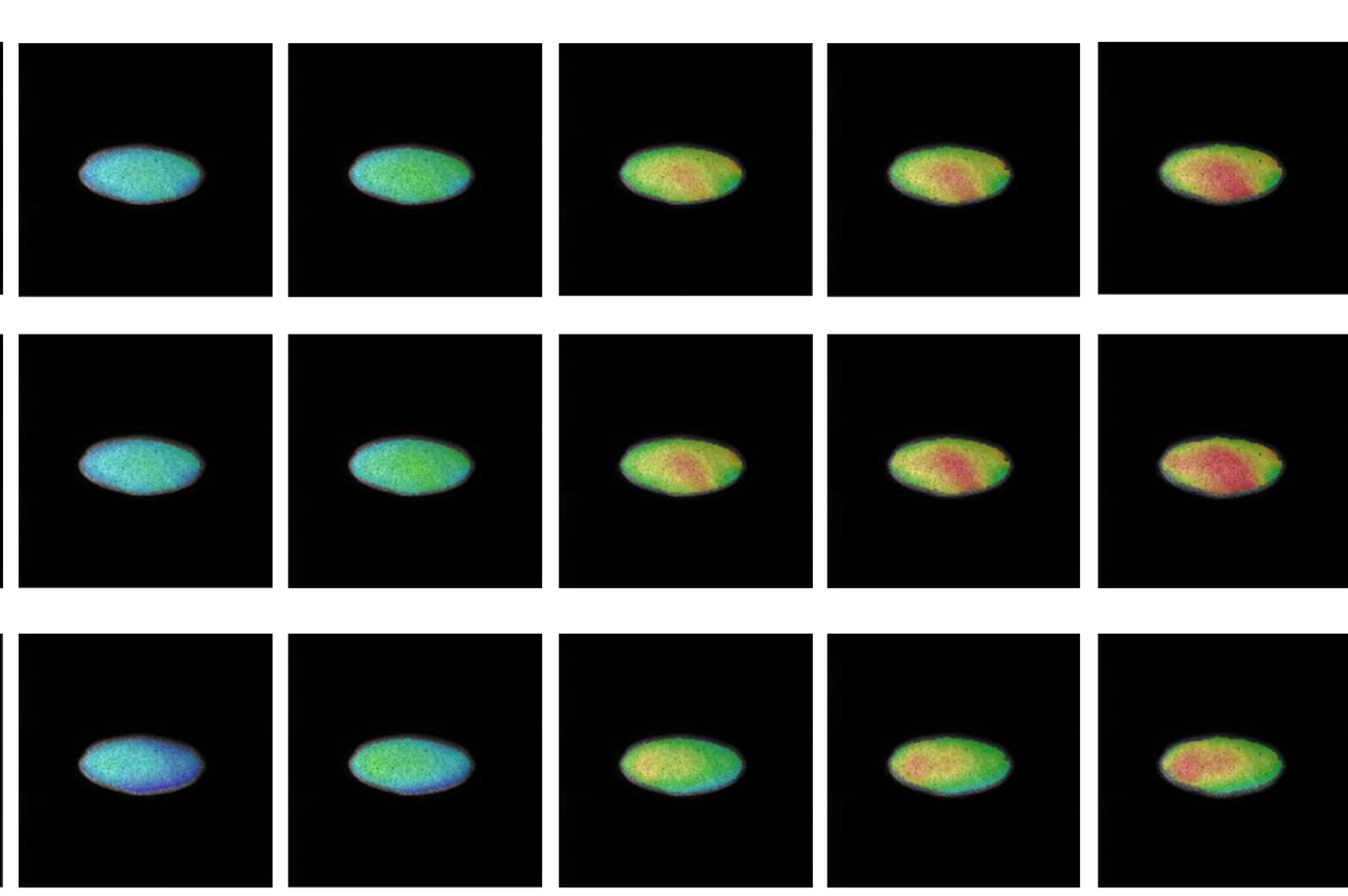
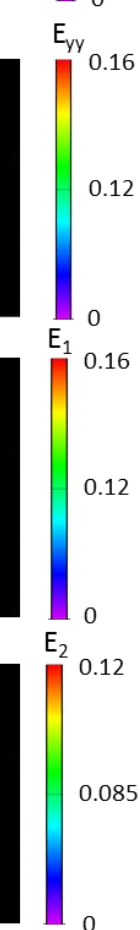
c)
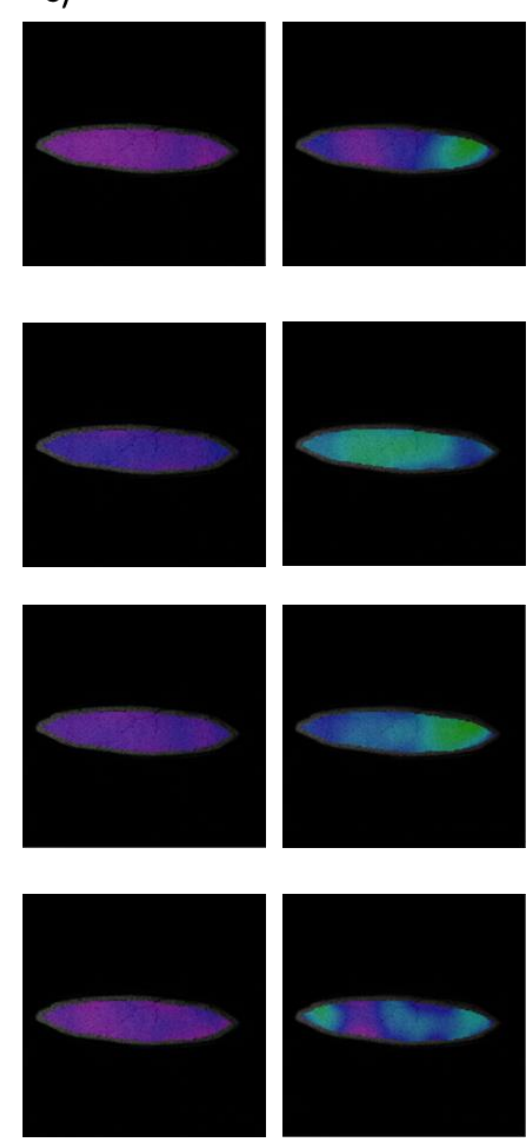

Figure 9
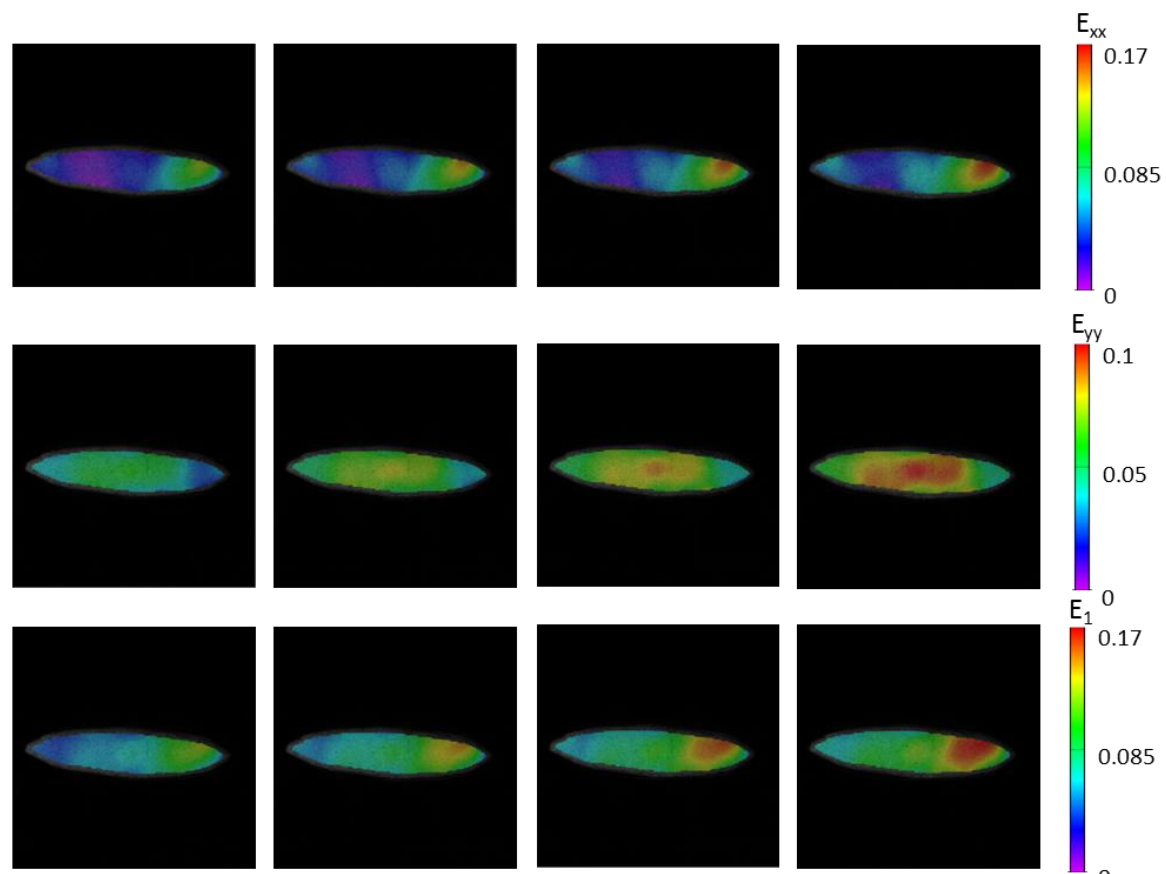

$\mathrm{E}_{1} 0$
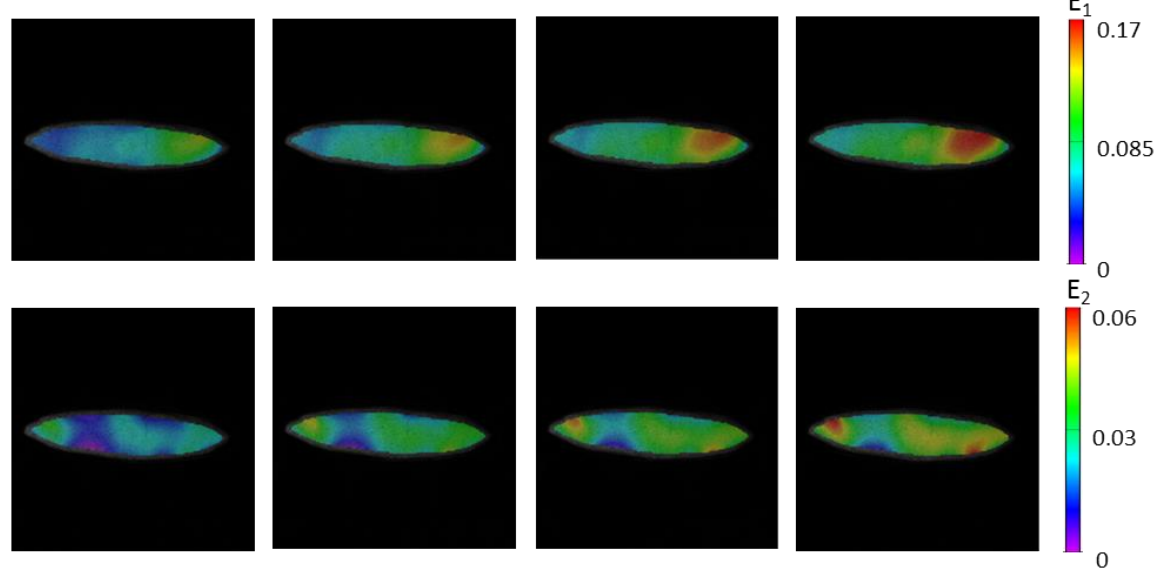